\title{
A Note on Translation, Transliteration, Names, and Measures
}

All translations are by the author unless otherwise indicated. Pinyin is used for the transliteration of Chinese terms. With the exception of Chinese writers who published in English and presented their names in Western fashion, Chinese personal names in the text appear in Chinese form, with surname preceding given name.

$1 m u=1 / 6$ acre

$1 \mathrm{jin}=1.1$ pounds; 1 dan $=100 \mathrm{jin}$

$1 \mathrm{li}=1 / 2$ kilometer $=0.31$ mile 